\title{
A Limited Memory BFGS Method for Solving Large-Scale Symmetric Nonlinear Equations
}

\author{
Xiangrong Li, Xiaoliang Wang, and Xiabin Duan \\ College of Mathematics and Information Science, Guangxi University, Nanning, Guangxi 530004, China \\ Correspondence should be addressed to Xiangrong Li; xrli68@163.com
}

Received 21 June 2014; Accepted 14 July 2014; Published 5 August 2014

Academic Editor: Gonglin Yuan

Copyright ( 2014 Xiangrong Li et al. This is an open access article distributed under the Creative Commons Attribution License, which permits unrestricted use, distribution, and reproduction in any medium, provided the original work is properly cited.

A limited memory BFGS (L-BFGS) algorithm is presented for solving large-scale symmetric nonlinear equations, where a line search technique without derivative information is used. The global convergence of the proposed algorithm is established under some suitable conditions. Numerical results show that the given method is competitive to those of the normal BFGS methods.

\section{Introduction}

Consider

$$
h(x)=0, \quad x \in \mathfrak{R}^{n},
$$

where $h: \mathfrak{R}^{n} \rightarrow \mathfrak{R}^{n}$ is continuously differentiable, the Jacobian $\nabla h(x)$ of $g$ is symmetric for all $x \in \Re^{n}$, and $n$ denotes the large-scale dimensions. It is not difficult to see that if $g$ is the gradient mapping of some function $f: \mathfrak{R}^{n} \rightarrow$ $\mathfrak{R}$, problem (1) is the first order necessary condition for the problem $\min _{x \in \Re^{n}} f(x)$. Furthermore, considering

$$
\min f(u) \quad \text { s.t. } a(u)=0 \text {, }
$$

where $a$ is a vector-valued function, then the KKT conditions can be represented as the system (1) with $x=(u, v)$ and $h(u, v)=(\nabla f(u)+\nabla a(u) v, a(u))$, where $v$ is the vector of Lagrange multipliers. The above two cases show that problem (1) can come from an unconstrained problem or an equality constrained optimization problem in theory. Moreover, there are other practical problems that can also take the form of (1), such as the discretized two-point boundary value problem, the saddle point problem, and the discretized elliptic boundary value problem (see Chapter 1 of [1] in detail). Let $\theta$ be the norm function $\theta(x)=(1 / 2)\|h(x)\|^{2}$; then problem (1) is equivalent to the following global optimization problem:

$$
\min \theta(x) \quad x \in \mathfrak{R}^{n},
$$

where $\|\cdot\|$ is the Euclidean norm.

In this paper we will focus on the line search method for (1), where its normal iterative formula is defined by

$$
x_{k+1}=x_{k}+\alpha_{k} d_{k},
$$

where $d_{k}$ is the so-called search direction and $\alpha_{k}$ is a steplength along $d_{k}$. To begin with, we briefly review some methods for $\alpha_{k}$.

(i) Normal Line Search (Brown and Saad [2]). The stepsize $\alpha_{k}$ is determined by

$$
\theta\left(x_{k}+\alpha_{k} d_{k}\right)-\theta\left(x_{k}\right) \leq \sigma \alpha_{k} \nabla \theta\left(x_{k}\right)^{T} d_{k},
$$

where $\sigma \in(0,1)$ and $\nabla \theta\left(x_{k}\right)=\nabla h\left(x_{k}\right) h\left(x_{k}\right)$. The convergence is proved and some good results are obtained. We all know that the nonmonotone idea is more interesting than the normal technique in many cases. Then a nonmonotone line search technique based on this motivation is presented by Zhu [3].

(ii) Nonmonotone Line Search (Zhu [3]). The stepsize $\alpha_{k}$ is determined by

$$
\theta\left(x_{k}+\alpha_{k} d_{k}\right)-\theta\left(x_{l(k)}\right) \leq \sigma \alpha_{k} \nabla \theta\left(x_{k}\right)^{T} d_{k},
$$


$\theta\left(x_{l(k)}\right)=\max _{0 \leq j \leq m(k)}\left\{\theta\left(x_{k-j}\right)\right\}, m(0)=0, m(k)=\min \{m(k-$ $1)+1, M\}$ (for $k \geq 1$ ), and $M$ is a nonnegative integer. The global convergence and the superlinear convergence are established under mild conditions, respectively. It is not difficult to see that, for the above two line search techniques, the Jacobian matrix $\nabla h_{k}$ must be computed at each iteration, which obviously increase the workload and the CPU time consumed. In order to avoid this drawback, Yuan and Lu [4] presented a new backtracking inexact technique.

(iii) A New Line Search (Yuan and Lu [4]). The stepsize $\alpha_{k}$ is determined by

$$
\left\|h\left(x_{k}+\alpha_{k} d_{k}\right)\right\|^{2} \leq\left\|h\left(x_{k}\right)\right\|^{2}+\delta \alpha_{k}^{2} h_{k}^{T} d_{k},
$$

where $\delta \in(0,1)$ and $h_{k}=h\left(x_{k}\right)$. They established the global convergence and the superlinear convergence. And the numerical tests showed that the new line search technique is more effective than those of the normal line search technique. However, these three line search techniques can not directly ensure the descent property of $d_{k}$. Thus more interesting line search techniques are studied.

(iv) Approximate Monotone Line Search (Li and Fukushima [5]). The stepsize $\alpha_{k}$ is determined by

$$
\begin{aligned}
\theta\left(x_{k}+\alpha_{k} d_{k}\right)-\theta\left(x_{k}\right) \leq & -\delta_{1}\left\|\alpha_{k} d_{k}\right\|^{2} \\
& -\delta_{2}\left\|\alpha_{k} h_{k}\right\|^{2}+\epsilon_{k}\left\|h_{k}\right\|^{2},
\end{aligned}
$$

where $\alpha_{k}=r^{i_{k}}, r \in(0,1), i_{k}$ is the smallest nonnegative integer $i$ satisfying (8), $\delta_{1}>0$ and $\delta_{2}>0$ are constants, and $\epsilon_{k}$ is such that

$$
\sum_{k=0}^{\infty} \epsilon_{k}<\infty
$$

The line search (8) can be rewritten as

$$
\begin{aligned}
\left\|h\left(x_{k}+\alpha d_{k}\right)\right\|^{2} \leq & \left(1+\epsilon_{k}\right)\left\|h_{k}\right\|^{2}-\delta_{1}\left\|\alpha h_{k}\right\|^{2} \\
& -\delta_{2}\left\|\alpha d_{k}\right\|^{2} ;
\end{aligned}
$$

it is straightforward to see that as $\alpha \rightarrow 0$, the right-hand side of the above inequality goes to $\left(1+\epsilon_{k}\right)\left\|h_{k}\right\|^{2}$. Then it is not difficult to see that the sequence $\left\{x_{k}\right\}$ generated by one algorithm with line search (8) is approximately norm descent. In order to ensure the sequence $\left\{x_{k}\right\}$ is norm descent, $\mathrm{Gu}$ et al. [6] presented the following line search.

(v) Monotone Descent Line Search (Gu et al. [6]). The stepsize $\alpha_{k}$ is determined by

$$
\begin{aligned}
\left\|h\left(x_{k}+\alpha_{k} d_{k}\right)\right\|^{2}-\left\|h_{k}\right\|^{2} \leq & -\delta_{1}\left\|\alpha_{k} h_{k}\right\|^{2} \\
& -\delta_{2}\left\|\alpha_{k} d_{k}\right\|^{2},
\end{aligned}
$$

where $\alpha_{k}, \delta_{1}$, and $\delta_{2}$ are similar to (8).

In the following, we present some techniques for $d_{k}$.

(i) Newton Method. The search direction $d_{k}$ is defined by

$$
\nabla h\left(x_{k}\right) d_{k}=-h\left(x_{k}\right) \text {. }
$$

Newton method is one of the most effective methods since it normally requires a fewest number of function evaluations and is very good at handling ill-conditioning. However, its efficiency largely depends on the possibility to efficiently solve a linear system (12) which arises when computing. Moreover, the exact solution of the system (12) could be too burdensome or is not necessary when $x_{k}$ is far from a solution [7]. Thus the quasi-Newton methods are proposed.

(ii) Quasi-Newton Method. The search direction $d_{k}$ is defined by

$$
B_{k} d_{k}+h_{k}=0
$$

where $B_{k}$ is the quasi-Newton update matrix. The quasiNewton methods represent the basic approach underlying most of the Newton-type large-scale algorithms (see $[3,4,8]$, etc.), where the famous BFGS method is one of the most effective quasi-Newton methods, generated by the following formula:

$$
B_{k+1}=B_{k}-\frac{B_{k} s_{k} s_{k}^{T} B_{k}}{s_{k}^{T} B_{k} s_{k}}+\frac{y_{k} y_{k}^{T}}{y_{k}^{T} s_{k}},
$$

where $s_{k}=x_{k+1}-x_{k}$ and $y_{k}=h_{k+1}-h_{k}$ with $h_{k}=h\left(x_{k}\right)$ and $h_{k+1}=h\left(x_{k}+\alpha_{k} d_{k}\right)$. By (11) and (14), Yuan and Yao [9] proposed a BFGS method for nonlinear equations and some good results were obtained. Denote $H_{k}=B_{k}^{-1}$, and then (14) has the inverse update formula represented by

$$
\begin{aligned}
H_{k+1}= & H_{k}-\frac{y_{k}^{T}\left(s_{k}-H_{k} y_{k}\right) s_{k} s_{k}^{T}}{\left(y_{k}^{T} s_{k}\right)^{2}} \\
& +\frac{\left(s_{k}-H_{k} y_{k}\right) s_{k}^{T}+s_{k}\left(s_{k}-H_{k} y_{k}\right)^{T}}{\left(y_{k}^{T} s_{k}\right)^{2}} \\
= & \left(I-\frac{s_{k} y_{k}^{T}}{y_{k}^{T} s_{k}}\right) H_{k}\left(I-\frac{y_{k} s_{k}^{T}}{y_{k}^{T} s_{k}}\right)+\frac{s_{k} s_{k}^{T}}{y_{k}^{T} s_{k}} .
\end{aligned}
$$

Unfortunately, both the Newton method and the quasiNewton method require many space to store $n \times n$ matrix at every iteration, which will prevent the efficiency of the algorithm for problems, especially for large-scale problems. Therefore low storage matrix information method should be necessary.

(iii) Limited Memory Quasi-Newton Method. The search direction $d_{k}$ is defined by

$$
d_{k}=-H_{k} h_{k}
$$

where $H_{k}$ is generated by limited memory quasi-Newton method, where the famous limited memory quasi-Newton method is the so-called limited memory BFGS method. The L-BFGS method is an adaptation of the BFGS method for large-scale problems (see [10] in detail), which often 
requires minimal storage and provides a fast rate of linear convergence. The L-BFGS method has the following form:

$$
\begin{aligned}
H_{k+1}= & V_{k}^{T} H_{k} V_{k}+\rho_{k} s_{k} s_{k}^{T} \\
= & V_{k}^{T}\left[V_{k-1}^{T} H_{k-1} V_{k-1}+\rho_{k-1} s_{k-1} s_{k-1}^{T}\right] V_{k}+\rho_{k} s_{k} s_{k}^{T} \\
= & \cdots \\
= & {\left[V_{k}^{T} \cdots V_{k-\widetilde{m}+1}^{T}\right] H_{k-\widetilde{m}+1}\left[V_{k-\widetilde{m}+1} \cdots V_{k}\right] } \\
& +\rho_{k-\widetilde{m}+1}\left[V_{k-1}^{T} \cdots V_{k-\widetilde{m}+2}^{T}\right] \\
& \times s_{k-\widetilde{m}+1} s_{k-\widetilde{m}+1}^{T}\left[V_{k-\widetilde{m}+2} \cdots V_{k-1}\right]+\cdots+\rho_{k} s_{k} s_{k}^{T},
\end{aligned}
$$

where $\rho_{k}=1 / s_{k}^{T} y_{k}, V_{k}=I-\rho_{k} y_{k} s_{k}^{T}, \widetilde{m}>0$ is an integer, and $I$ is the unit matrix. Formula (17) shows that matrix $H_{k}$ is obtained by updating the basic matrix $H_{0} \widetilde{m}$ times using BFGS formula with the previous $\widetilde{m}$ iterations. By (17), together with (7) and (8), Yuan et al. [11] presented the L-BFGS method for nonlinear equations and got the global convergence. At present, there are many papers proposed for (1) (see $[6,12-$ 15], etc.).

In order to effectively solve large-scale nonlinear equations and possess good theory property, based on the above discussions of $\alpha_{k}$ and $d_{k}$, we will combine (11) and (16) and present a L-BFGS method for (1) since (11) can make the norm function be descent and (16) need less low storage. The main attributes of the new algorithm are stated as follows.

(i) A L-BFGS method with (11) is presented.

(ii) The norm function is descent.

(iii) The global convergence is established under appropriate conditions.

(iv) Numerical results show that the given algorithm is more competitive than the normal algorithm for large-scale nonlinear equations.

This paper is organized as follows. In the next section, the backtracking inexact L-BFGS algorithm is stated. Section 3 will present the global convergence of the algorithm under some reasonable conditions. Numerical experiments are done to test the performance of the algorithms in Section 4.

\section{Algorithms}

This section will state the L-BFGS method in association with the new backtracking line search technique (11) for solving (1).

\section{Algorithm 1.}

Step 0. Choose an initial point $x_{0} \in \mathfrak{R}^{n}$, an initial symmetric positive definite matrix $H_{0} \in \Re^{n \times n}$, positive constants $\delta_{1}, \delta_{2}$, constants $r, \rho \in(0,1)$, and a positive integer $m_{1}$. Let $k:=0$.

Step 1. Stop if $\left\|h_{k}\right\|=0$.

Step 2. Determine $d_{k}$ by (16).
Step 3. If

$$
\left\|h\left(x_{k}+d_{k}\right)\right\| \leq \rho\left\|h\left(x_{k}\right)\right\|,
$$

then take $\alpha_{k}=1$ and go to Step 5. Otherwise go to Step 4 .

Step 4. Let $i_{k}$ be the smallest nonnegative integer $i$ such that (11) holds for $\alpha=r^{i}$. Let $\alpha_{k}=r^{i_{k}}$.

Step 5. Let the next iterative be $x_{k+1}=x_{k}+\alpha_{k} d_{k}$.

Step 6. Let $\widetilde{m}=\min \left\{k+1, m_{1}\right\}$. Put $s_{k}=x_{k+1}-x_{k}=\alpha_{k} d_{k}$ and $y_{k}=h_{k+1}-h_{k}$. Update $H_{0}$ for $\widetilde{m}$ times to get $H_{k+1}$ by (17).

Step 7. Let $k:=k+1$. Go to Step 1 .

In the following, to conveniently analyze the global convergence, we assume that the algorithm updates $B_{k}$ (the inverse of $H_{k}$ ) with the basically bounded and positive definite matrix $B_{0}\left(H_{0}\right.$ 's inverse). Then Algorithm 1 with $B_{k}$ has the following steps.

Algorithm 2.

Step 2. Determine $d_{k}$ by

$$
B_{k} d_{k}=-h_{k}
$$

Step 6. Let $\widetilde{m}=\min \left\{k+1, m_{1}\right\}$. Put $s_{k}=x_{k+1}-x_{k}=\alpha_{k} d_{k}$ and $y_{k}=h_{k+1}-h_{k}$. Update $B_{0}$ for $\widetilde{m}$ times; that is, for $l=$ $k-\widetilde{m}+1, \ldots, k$ compute

$$
B_{k}^{l+1}=B_{k}^{l}-\frac{B_{k}^{l} s_{l} s_{l}^{T} B_{k}^{l}}{s_{l}^{T} B_{k}^{l} s_{l}}+\frac{y_{l} y_{l}^{T}}{y_{l}^{T} s_{l}},
$$

where $s_{l}=x_{l+1}-x_{l}, y_{l}=h_{l+1}-h_{l}$, and $B_{k}^{k-\widetilde{m}+1}=B_{0}$ for all $k$.

Remark 3. Algorithms 1 and 2 are mathematically equivalent. Throughout this paper, Algorithm 2 is given only for the purpose of analysis, so we only discuss Algorithm 2 in theory. In the experiments, we implement Algorithm 1.

\section{Global Convergence}

Define the level set $\Omega$ by

$$
\Omega=\left\{x \mid\|h(x)\| \leq\left\|h\left(x_{0}\right)\right\|\right\} .
$$

In order to establish the global convergence of Algorithm 2, similar to $[4,11]$, we need the following assumptions.

Assumption A. $g$ is continuously differentiable on an open convex set $\Omega_{1}$ containing $\Omega$. Moreover the Jacobian of $g$ is symmetric, bounded, and positive definite on $\Omega_{1}$; namely, there exist positive constants $M \geq m>0$ satisfying

$$
\begin{gathered}
\|\nabla h(x)\| \leq M \quad \forall x \in \Omega_{1}, \\
m\|d\|^{2} \leq d^{T} \nabla h(x) d \quad \forall x \in \Omega_{1}, d \in \Re^{n} .
\end{gathered}
$$


Assumption B. $B_{k}$ is a good approximation to $\nabla g_{k}$; that is,

$$
\left\|\left(\nabla h_{k}-B_{k}\right) d_{k}\right\| \leq \epsilon\left\|h_{k}\right\|
$$

where $\epsilon \in(0,1)$ is a small quantity.

Remark 4. Assumption A implies

$$
\left\|y_{k}\right\| \leq M\left\|s_{k}\right\|, \quad y_{k}^{T} s_{k} \geq m\left\|s_{k}\right\|^{2} .
$$

The relations in (24) can ensure that $B_{k+1}$ generated by (20) inherits symmetric and positive definiteness of $B_{k}$. Thus, (19) has a unique solution for each $k$. Moreover, the following lemma holds.

Lemma 5 (see Theorem 2.1 in [16] or see Lemma 3.4 of [11]). Let Assumption A hold and let $\left\{\alpha_{k}, d_{k}, x_{k+1}, g_{k+1}\right\}$ be generated by Algorithm 2. Then, for any $r_{0} \in(0,1)$ and $k \geq 0$, there are positive constants $\beta_{j}, j=1,2,3$; the following relations

$$
\beta_{2}\left\|s_{i}\right\|^{2} \leq s_{i}^{T} B_{i} s_{i} \leq \beta_{3}\left\|s_{i}\right\|^{2}, \quad\left\|B_{i} s_{i}\right\| \leq \beta_{1}\left\|s_{i}\right\|
$$

hold for at least $\left\lceil r_{0} k\right\rceil$ values of $i \in[0, k]$.

By Assumption B, similar to $[4,9,11,15]$, it is easy to get the following lemma.

Lemma 6. Let Assumption B hold and let $\left\{\alpha_{k}, d_{k}, x_{k+1}, h_{k+1}\right\}$ be generated by Algorithm 2. Then $d_{k}$ is a descent direction for $\theta(x)$ at $x_{k}$; that is, $\nabla \theta\left(x_{k}\right)^{T} d_{k}<0$ holds.

Based on the above lemma, by Assumption B, similar to Lemma 3.8 in [2], we can get the following lemma.

Lemma 7. Let Assumption B hold and let $\left\{\alpha_{k}, d_{k}, x_{k+1}, h_{k+1}\right\}$ be generated by Algorithm 2. Then $\left\{x_{k}\right\} \subset \Omega$. Moreover, $\left\{\left\|h_{k}\right\|\right\}$ converges.

Lemma 8. Let Assumptions $A$ and $B$ hold. Then, in a finite number of backtracking steps, Algorithm 2 will produce an iterate $x_{k+1}=x_{k}+\alpha_{k} d_{k}$.

Proof. It is sufficient for us to prove that the line search (11) is reasonable. By Lemma 3.8 in [2], we can deduce that, in a finite number of backtracking steps, $\alpha_{k}$ is such that

$$
\begin{array}{r}
\left\|h\left(x_{k}+\alpha_{k} d_{k}\right)\right\|^{2}-\left\|h\left(x_{k}\right)\right\|^{2} \leq \delta \alpha_{k} h\left(x_{k}\right)^{T} \nabla h\left(x_{k}\right) d_{k}, \\
\delta \in(0,1) .
\end{array}
$$

By (19), we get

$$
\begin{aligned}
\nabla \theta\left(x_{k}\right)^{T} d_{k} & =h\left(x_{k}\right)^{T} \nabla h\left(x_{k}\right) d_{k} \\
& =h\left(x_{k}\right)^{T}\left[\left(\nabla h\left(x_{k}\right)-B_{k}\right) d_{k}-h\left(x_{k}\right)\right] \\
& =h\left(x_{k}\right)^{T}\left(\nabla h\left(x_{k}\right)-B_{k}\right) d_{k}-h\left(x_{k}\right)^{T} h\left(x_{k}\right) .
\end{aligned}
$$

Thus

$$
\begin{aligned}
\nabla \theta\left(x_{k}\right)^{T} d_{k}+\left\|h_{k}\right\|^{2} & \leq h\left(x_{k}\right)^{T}\left(\nabla h\left(x_{k}\right)-B_{k}\right) d_{k} \\
& \leq\left\|h\left(x_{k}\right)\right\|\left\|\left(\nabla h\left(x_{k}\right)-B_{k}\right) d_{k}\right\| .
\end{aligned}
$$

By Assumption B, we have

$$
\begin{aligned}
\nabla \theta\left(x_{k}\right)^{T} d_{k} & \leq\left\|h\left(x_{k}\right)\right\|\left\|\left(\nabla h\left(x_{k}\right)-B_{k}\right) d_{k}\right\|-\left\|h\left(x_{k}\right)\right\|^{2} \\
& \leq-(1-\epsilon)\left\|h\left(x_{k}\right)\right\|^{2} .
\end{aligned}
$$

Using (19) again and $\alpha_{k} \leq 1$, we obtain

$$
\begin{aligned}
\alpha_{k} h\left(x_{k}\right)^{T} \nabla h\left(x_{k}\right) d_{k} & \leq-\alpha_{k}(1-\epsilon)\left\|h\left(x_{k}\right)\right\|^{2} \\
& =-\frac{(1-\epsilon)}{2 \alpha_{k}}\left\|\alpha_{k} h_{k}\right\|^{2}-\frac{(1-\epsilon)}{2 \alpha_{k}}\left\|\alpha_{k} B_{k} d_{k}\right\|^{2} \\
& \leq-\frac{(1-\epsilon)}{2}\left\|\alpha_{k} h_{k}\right\|^{2}-\frac{\beta_{2}^{2}(1-\epsilon)}{2}\left\|\alpha_{k} d_{k}\right\|^{2} .
\end{aligned}
$$

Setting $\delta_{1} \in(0, \delta((1-\epsilon) / 2))$ and $\delta_{2} \in\left(0, \delta\left(\beta_{2}^{2}(1-\epsilon) / 2\right)\right)$ implies (11). This completes the proof.

Remark 9. The above lemma shows that Algorithm 2 is well defined. By a way similar to Lemma 3.2 and Corollary 3.4 in [5], it is not difficult to deduce that

$$
\alpha_{k} \geq \frac{\beta_{2} r}{\beta_{1}^{2} \sigma_{1}+\sigma_{2}+M^{2}}
$$

holds; we do not prove it anymore. Now we establish the global convergence theorem.

Theorem 10. Let Assumptions $A$ and $B$ hold. Then the sequence $\left\{x_{k}\right\}$ generated by Algorithm 2 converges to the unique solution $x^{*}$ of (1).

Proof. Lemma 7 implies that $\left\{\left\|h_{k}\right\|\right\}$ converges. If

$$
\lim _{k \rightarrow \infty}\left\|h_{k}\right\|=0
$$

then every accumulation point of $\left\{x_{k}\right\}$ is a solution of (1). Assumption A means that (1) has only one solution. Moreover, since $\Omega$ is bounded, $\left\{x_{k}\right\} \subseteq \Omega$ has at least one accumulation point. Therefore $\left\{x_{k}\right\}$ itself converges to the unique solution of (1). Therefore, it suffices to verify (32).

If (18) holds for infinitely many $k$ 's, then (32) is trivial. Otherwise, if (18) holds for only finitely many $k$ 's, we conclude that Step 3 is executed for all $k$ sufficiently large. By (11), we have

$$
\delta_{1}\left\|\alpha_{k} h_{k}\right\|^{2}+\delta_{2}\left\|s_{k}\right\|^{2} \leq\left\|h_{k}\right\|^{2}-\left\|h_{k+1}\right\|^{2} .
$$

Since $\left\{\left\|h_{k}\right\|\right\}$ is bounded, by adding these inequalities, we get

$$
\sum_{k=0}^{\infty}\left\|\alpha_{k} h_{k}\right\|^{2}<\infty, \quad \sum_{k=0}^{\infty}\left\|\alpha_{k} d_{k}\right\|^{2}<\infty .
$$

Then we have

$$
\lim _{k \rightarrow \infty}\left\|\alpha_{k} h_{k}\right\|=0,
$$

which together with (31) implies (32). This completes the proof. 


\section{Numerical Results}

This section reports numerical results with Algorithm 1 and normal BFGS algorithm. The test problems with the associated initial guess $x_{0}$ are listed with

$$
h(x)=\left(f_{1}(x), f_{2}(x), \ldots, f_{n}(x)\right)^{T} .
$$

Problem 1. Exponential function 1:

$$
\begin{gathered}
f_{1}(x)=e^{x_{1}-1}-1, \\
f_{i}(x)=i\left(e^{x_{i}-1}-x_{i}\right), \quad i=2,3, \ldots, n .
\end{gathered}
$$

Initial guess: $x_{0}=\left(1 / n^{2}, 1 / n^{2}, \ldots, 1 / n^{2}\right)^{T}$.

Problem 2. Exponential function 2:

$$
\begin{gathered}
f_{1}(x)=e^{x_{1}}-1, \\
f_{i}(x)=\frac{i}{10}\left(e^{x_{i}}+x_{i-1}-i\right), \quad i=2,3, \ldots, n .
\end{gathered}
$$

Initial guess: $x_{0}=\left(1 / n^{2}, 1 / n^{2}, \ldots, 1 / n^{2}\right)^{T}$.

Problem 3. Trigonometric function:

$$
\begin{aligned}
f_{i}(x)= & 2\left(n+i\left(1-\cos x_{i}\right)-\sin x_{i}-\sum_{j=1}^{n} \cos x_{j}\right) \\
& \times\left(2 \sin x_{i}-\cos x_{i}\right), \quad i=1,2,3, \ldots, n .
\end{aligned}
$$

Initial guess: $x_{0}=(101 / 100 n, 101 / 100 n, \ldots, 101 / 100 n)^{T}$.

Problem 4. Singular function:

$$
\begin{gathered}
f_{1}(x)=\frac{1}{3} x_{1}^{3}+\frac{1}{2} x_{2}^{2}, \\
f_{i}(x)=-\frac{1}{2} x_{i}^{2}+\frac{i}{3} x_{i}^{3}+\frac{1}{2} x_{i+1}^{2}, \quad i=2,3, \ldots, n-1, \\
f_{n}(x)=-\frac{1}{2} x_{n}^{2}+\frac{n}{3} x_{n}^{3} .
\end{gathered}
$$

Initial guess: $x_{0}=(1,1, \ldots, 1)^{T}$.

Problem 5. Logarithmic function:

$$
f_{i}(x)=\ln \left(x_{i}+1\right)-\frac{x_{i}}{n}, \quad i=1,2,3, \ldots, n .
$$

Initial guess: $x_{0}=(1,1, \ldots, 1)^{T}$.

Problem 6. Broyden tridiagonal function [17, pages 471-472]:

$$
\begin{gathered}
f_{1}(x)=\left(3-0.5 x_{1}\right) x_{1}-2 x_{2}+1, \\
f_{i}(x)=\left(3-0.5 x_{i}\right) x_{i}-x_{i-1}+2 x_{i+1}+1, \\
i=2,3, \ldots, n-1, \\
f_{n}(x)=\left(3-0.5 x_{n}\right) x_{n}-x_{n-1}+1 .
\end{gathered}
$$

Initial guess: $x_{0}=(-1,-1, \ldots,-1)^{T}$.
Problem 7. Trigexp function [17, page 473]:

$$
\begin{gathered}
f_{1}(x)=3 x_{1}^{3}+2 x_{2}-5+\sin \left(x_{1}-x_{2}\right) \sin \left(x_{1}+x_{2}\right), \\
f_{i}(x)=-x_{i-1} e^{x_{i-1}-x_{i}}+x_{i}\left(4+3 x_{i}^{2}\right)+2 x_{i+1} \\
+\sin \left(x_{i}-x_{i+1}\right) \sin \left(x_{i}+x_{i+1}\right)-8, \\
i=2,3, \ldots, n-1, \\
f_{n}(x)=-x_{n-1} e^{x_{n-1}-x_{n}}+4 x_{n}-3 .
\end{gathered}
$$

Initial guess: $x_{0}=(0,0, \ldots, 0)^{T}$.

Problem 8. Strictly convex function 1 [18, page 29]: $h(x)$ is the gradient of $h(x)=\sum_{i=1}^{n}\left(e^{x_{i}}-x_{i}\right)$. Consider

$$
f_{i}(x)=e^{x_{i}}-1, \quad i=1,2,3, \ldots, n .
$$

Initial guess: $x_{0}=(1 / n, 2 / n, \ldots, 1)^{T}$.

Problem 9. Linear function-full rank:

$$
f_{i}(x)=x_{i}-\frac{2}{n} \sum_{j=1}^{n} x_{j}+1 .
$$

Initial guess: $x_{0}=(100,100, \ldots, 100)^{T}$.

Problem 10. Penalty function:

$$
\begin{gathered}
f_{i}(x)=\sqrt{10^{-5}}\left(x_{i}-1\right), \quad i=1,2,3, \ldots, n-1, \\
f_{n}(x)=\left(\frac{1}{4 n}\right) \sum_{j=1}^{n} x_{j}^{2}-\frac{1}{4} .
\end{gathered}
$$

Initial guess: $x_{0}=(1 / 3,1 / 3, \ldots, 1 / 3)^{T}$.

Problem 11. Variable dimensioned function:

$$
\begin{gathered}
f_{i}(x)=x_{i}-1, \quad i=1,2,3, \ldots, n-2, \\
f_{n-1}(x)=\sum_{j=1}^{n-2} j\left(x_{j}-1\right), \\
f_{n}(x)=\left(\sum_{j=1}^{n-2} j\left(x_{j}-1\right)\right)^{2} .
\end{gathered}
$$

Initial guess: $x_{0}=(1-(1 / n), 1-(2 / n), \ldots, 0)^{T}$.

Problem 12. Tridiagonal system [19]:

$$
\begin{gathered}
f_{1}(x)=4\left(x_{1}-x_{2}^{2}\right), \\
f_{i}(x)=8 x_{i}\left(x_{i}^{2}-x_{i-1}\right)-2\left(1-x_{i}\right) \\
+4\left(x_{i}-x_{i+1}^{2}\right), \quad i=2,3, \ldots, n-1, \\
f_{n}(x)=8 x_{n}\left(x_{n}^{2}-x_{n-1}\right)-2\left(1-x_{n}\right) .
\end{gathered}
$$

Initial guess: $x_{0}=(12,12, \ldots, 12)$. 
TABle

\begin{tabular}{|c|c|c|c|c|c|c|c|}
\hline \multirow{2}{*}{ Nr. } & \multirow{2}{*}{ Dim } & \multicolumn{3}{|c|}{ Algorithm 1} & \multicolumn{3}{|c|}{ Normal BFGS algorithm } \\
\hline & & $\mathrm{NI} / \mathrm{NG}$ & GN & Time & $\mathrm{NI} / \mathrm{NG}$ & $\mathrm{GN}$ & Time \\
\hline \multirow{4}{*}{ Problem 1} & 500 & $24 / 25$ & $9.908338 e-05$ & $2.901619 e+01$ & $23 / 87$ & $\mathrm{NaN}$ & $1.950013 e+00$ \\
\hline & 1000 & $9 / 10$ & $5.697414 e-05$ & $5.366434 e+01$ & $23 / 87$ & $\mathrm{NaN}$ & $1.124767 e+01$ \\
\hline & 1500 & $9 / 10$ & $3.363290 e-05$ & $1.689803 e+02$ & $\mathrm{NI}>1000$ & Inf & $8.094892 e+01$ \\
\hline & 2000 & $9 / 10$ & $2.706880 e-05$ & $3.880213 e+02$ & $\mathrm{NI}>1000$ & Inf & $1.550962 e+02$ \\
\hline \multirow{4}{*}{ Problem 2} & 500 & $8 / 16$ & $3.660478 e-05$ & $6.926444 e+00$ & $60 / 390$ & $\mathrm{NaN}$ & $7.269647 e+00$ \\
\hline & 1000 & $8 / 16$ & $6.406401 e-05$ & $4.513109 e+01$ & $40 / 314$ & $\mathrm{NaN}$ & $2.857938 e+01$ \\
\hline & 1500 & $9 / 17$ & $7.655321 e-05$ & $1.689959 e+02$ & $33 / 258$ & $\mathrm{NaN}$ & $7.102726 e+01$ \\
\hline & 2000 & $9 / 17$ & $9.651851 e-05$ & $3.881617 e+02$ & $29 / 226$ & $\mathrm{NaN}$ & $1.410717 e+02$ \\
\hline \multirow{4}{*}{ Problem 3} & 500 & $18 / 33$ & $7.747640 e-06$ & $2.110694 e+01$ & $\mathrm{NI}>1000$ & $5.905028 e+04$ & $1.220396 e+02$ \\
\hline & 1000 & $17 / 32$ & $8.381440 e-05$ & $1.270472 e+02$ & $\mathrm{NI}>1000$ & $1.577282 e+05$ & $5.830069 e+02$ \\
\hline & 1500 & $17 / 32$ & $6.658002 e-05$ & $3.975217 e+02$ & $\mathrm{NI}>1000$ & $2.929920 e+05$ & $1.645561 e+03$ \\
\hline & 2000 & $17 / 32$ & $5.689277 e-05$ & $9.154451 e+02$ & $\mathrm{NI}>1000$ & $4.345902 e+05$ & $3.776363 e+03$ \\
\hline \multirow{4}{*}{ Problem 4} & 500 & $809 / 3134$ & $9.614778 e-05$ & $1.113785 e+03$ & $9 / 73$ & $\mathrm{NaN}$ & $1.107607 e+00$ \\
\hline & 1000 & $960 / 3894$ & $9.865549 e-05$ & $8.674373 e+03$ & $8 / 65$ & $\mathrm{NaN}$ & $5.725237 e+00$ \\
\hline & 1500 & $197 / 695$ & $8.562547 e-05$ & $5.509394 e+03$ & $8 / 65$ & $\mathrm{NaN}$ & $1.584970 e+01$ \\
\hline & 2000 & $220 / 676$ & $9.847745 e-05$ & $1.418851 e+04$ & $8 / 65$ & $\mathrm{NaN}$ & $3.584903 e+01$ \\
\hline \multirow{4}{*}{ Problem 5} & 500 & $6 / 7$ & $4.925073 e-06$ & $4.305628 e+00$ & $6 / 7$ & $4.925073 e-06$ & $7.488048 e-01$ \\
\hline & 1000 & $6 / 7$ & $6.747222 e-06$ & $2.725337 e+01$ & $6 / 7$ & $6.747222 e-06$ & $4.477229 e+00$ \\
\hline & 1500 & $6 / 7$ & $8.176417 e-06$ & $8.352294 e+01$ & $6 / 7$ & $8.176417 e-06$ & $1.340049 e+01$ \\
\hline & 2000 & $6 / 7$ & $9.391335 e-06$ & $1.925364 e+02$ & $6 / 7$ & $9.391335 e-06$ & $3.001459 e+01$ \\
\hline \multirow{4}{*}{ Problem 6} & 500 & $96 / 97$ & $9.183793 e-05$ & $1.272812 e+02$ & $65 / 66$ & $9.473359 e-05$ & $8.346053 e+00$ \\
\hline & 1000 & $17 / 18$ & $7.206980 e-05$ & $1.263140 e+02$ & $63 / 64$ & $8.700852 e-05$ & $4.623870 e+01$ \\
\hline & 1500 & $17 / 18$ & $6.663689 e-05$ & $3.956965 e+02$ & $63 / 64$ & $9.605270 e-05$ & $1.402917 e+02$ \\
\hline & 2000 & $17 / 18$ & $6.136239 e-05$ & $9.107026 e+02$ & $64 / 65$ & $8.724048 e-05$ & $3.194588 e+02$ \\
\hline \multirow{4}{*}{ Problem 7} & 500 & $14 / 15$ & $7.551200 e-05$ & $1.533490 e+01$ & $52 / 53$ & $9.761790 e-05$ & $6.333641 e+00$ \\
\hline & 1000 & $15 / 16$ & $5.530847 e-05$ & $1.084051 e+02$ & $55 / 63$ & $8.871231 e-05$ & $3.985826 e+01$ \\
\hline & 1500 & $14 / 15$ & $8.445286 e-05$ & $3.119708 e+02$ & $60 / 68$ & $7.722045 e-05$ & $1.336617 e+02$ \\
\hline & 2000 & $15 / 16$ & $4.705617 e-05$ & $7.805354 e+02$ & $13 / 63$ & $\mathrm{NaN}$ & $5.564556 e+01$ \\
\hline \multirow{4}{*}{ Problem 8} & 500 & $6 / 7$ & $4.600878 e-05$ & $4.196427 e+00$ & $6 / 7$ & $2.375490 e-05$ & $7.020045 e-01$ \\
\hline & 1000 & $6 / 7$ & $6.434846 e-05$ & $2.658257 e+01$ & $6 / 7$ & $3.327487 e-05$ & $4.461629 e+00$ \\
\hline & 1500 & $6 / 7$ & $7.851881 e-05$ & $8.319533 e+01$ & $6 / 7$ & $4.062329 e-05$ & $1.335369 e+01$ \\
\hline & 2000 & $6 / 7$ & $9.049762 e-05$ & $1.928484 e+02$ & $6 / 7$ & $4.683284 e-05$ & $2.985859 e+01$ \\
\hline \multirow{4}{*}{ Problem 9} & 500 & $2 / 10$ & $2.027911 e-10$ & $5.460035 e-01$ & $\mathrm{NI}>1000$ & $4.514037 e+117$ & $1.037407 e+01$ \\
\hline & 1000 & $2 / 10$ & $2.666034 e-10$ & $2.698817 e+00$ & $\mathrm{NI}>1000$ & $6.383813 e+117$ & $2.861058 e+01$ \\
\hline & 1500 & $2 / 10$ & $1.379742 e-09$ & $8.330453 e+00$ & $\mathrm{NI}>1000$ & $7.818542 e+117$ & $5.319634 e+01$ \\
\hline & 2000 & $2 / 10$ & $1.701735 e-09$ & $1.942212 e+01$ & $\mathrm{NI}>1000$ & $9.028075 e+117$ & $8.375694 e+01$ \\
\hline \multirow{4}{*}{ Problem 10} & 500 & $435 / 2865$ & $5.154286 e-05$ & $5.901362 e+02$ & $41 / 210$ & $\mathrm{NaN}$ & $3.806424 e+00$ \\
\hline & 1000 & $637 / 4215$ & $5.701191 e-05$ & $5.749432 e+03$ & $67 / 425$ & $\mathrm{NaN}$ & $3.689424 e+01$ \\
\hline & 1500 & $303 / 1914$ & $4.003848 e-05$ & $8.526469 e+03$ & $63 / 491$ & $\mathrm{NaN}$ & $1.146919 e+02$ \\
\hline & 2000 & $473 / 3281$ & $3.558910 e-05$ & $3.074095 e+04$ & $64 / 499$ & $\mathrm{NaN}$ & $2.622845 e+02$ \\
\hline \multirow{4}{*}{ Problem 11} & 500 & $1 / 2$ & $0.000000 e+00$ & $1.092007 e-01$ & $1 / 2$ & $0.000000 e+00$ & $1.560010 e-01$ \\
\hline & 1000 & $1 / 2$ & $0.000000 e+00$ & $7.956051 e-01$ & $1 / 2$ & $0.000000 e+00$ & $6.864044 e-01$ \\
\hline & 1500 & $1 / 2$ & $0.000000 e+00$ & $2.277615 e+00$ & $1 / 2$ & $0.000000 e+00$ & $2.324415 e+00$ \\
\hline & 2000 & $1 / 2$ & $0.000000 e+00$ & $5.101233 e+00$ & $1 / 2$ & $0.000000 e+00$ & $5.210433 e+00$ \\
\hline \multirow{4}{*}{ Problem 12} & 500 & $260 / 800$ & $1.654459 e-05$ & $3.509398 e+02$ & $8 / 51$ & $\mathrm{NaN}$ & $7.800050 e-01$ \\
\hline & 1000 & $324 / 1053$ & $8.997051 e-05$ & $2.902289 e+03$ & $8 / 51$ & $\mathrm{NaN}$ & $5.226033 e+00$ \\
\hline & 1500 & $254 / 829$ & $1.257117 e-05$ & $7.123224 e+03$ & $8 / 51$ & $\mathrm{NaN}$ & $1.583410 e+01$ \\
\hline & 2000 & $372 / 1353$ & $9.331122 e-05$ & $2.414117 e+04$ & $8 / 51$ & $\mathrm{NaN}$ & $3.046700 e+01$ \\
\hline
\end{tabular}


TABle 1: Continued.

\begin{tabular}{|c|c|c|c|c|c|c|c|}
\hline \multirow{2}{*}{ Nr. } & \multirow{2}{*}{ Dim } & \multicolumn{3}{|c|}{ Algorithm 1} & \multicolumn{3}{|c|}{ Normal BFGS algorithm } \\
\hline & & $\mathrm{NI} / \mathrm{NG}$ & GN & Time & $\mathrm{NI} / \mathrm{NG}$ & $\mathrm{GN}$ & Time \\
\hline \multirow{4}{*}{ Problem 13} & 500 & $96 / 209$ & $9.194537 e-05$ & $1.287632 e+02$ & $10 / 74$ & $\mathrm{NaN}$ & $1.185608 e+00$ \\
\hline & 1000 & $53 / 89$ & $5.718492 e-05$ & $4.531049 e+02$ & $10 / 74$ & $\mathrm{NaN}$ & $6.021639 e+00$ \\
\hline & 1500 & $\mathrm{NI}>1000$ & $1.180195 e+10$ & $2.835008 e+04$ & $10 / 74$ & $\mathrm{NaN}$ & $1.790891 e+01$ \\
\hline & 2000 & $54 / 132$ & $3.919816 e-05$ & $3.337298 e+03$ & $10 / 74$ & $\mathrm{NaN}$ & $4.031066 e+01$ \\
\hline \multirow{4}{*}{ Problem 14} & 500 & $18 / 68$ & $5.826508 e-05$ & $2.076373 e+01$ & $16 / 115$ & $\mathrm{NaN}$ & $6.240040 e-01$ \\
\hline & 1000 & $18 / 68$ & $8.239959 e-05$ & $1.357989 e+02$ & $16 / 115$ & $\mathrm{NaN}$ & $3.354021 e+00$ \\
\hline & 1500 & $19 / 69$ & $5.002681 e-05$ & $4.532921 e+02$ & $16 / 115$ & $\mathrm{NaN}$ & $9.765663 e+00$ \\
\hline & 2000 & $19 / 69$ & $5.774900 e-05$ & $1.042820 e+03$ & $16 / 115$ & $\mathrm{NaN}$ & $2.148134 e+01$ \\
\hline \multirow{4}{*}{ Problem 15} & 500 & $8 / 9$ & $6.870902 e-05$ & $6.910844 e+00$ & $22 / 23$ & $8.899350 e-05$ & $2.667617 e+00$ \\
\hline & 1000 & $7 / 8$ & $7.203786 e-05$ & $3.561503 e+01$ & $16 / 17$ & $9.828207 e-05$ & $1.174688 e+01$ \\
\hline & 1500 & $7 / 8$ & $4.809670 e-05$ & $1.122739 e+02$ & $13 / 14$ & $9.654107 e-05$ & $2.903179 e+01$ \\
\hline & 2000 & $7 / 8$ & $3.609946 e-05$ & $2.583845 e+02$ & $11 / 12$ & $9.465949 e-05$ & $5.519315 e+01$ \\
\hline \multirow{4}{*}{ Problem 16} & 500 & $0 / 1$ & $0.000000 e+00$ & $0.000000 e+00$ & $0 / 1$ & $0.000000 e+00$ & $0.000000 e+00$ \\
\hline & 1000 & $0 / 1$ & $0.000000 e+00$ & $0.000000 e+00$ & $0 / 1$ & $0.000000 e+00$ & $0.000000 e+00$ \\
\hline & 1500 & $0 / 1$ & $0.000000 e+00$ & $1.560010 e-02$ & $0 / 1$ & $0.000000 e+00$ & $0.000000 e+00$ \\
\hline & 2000 & $0 / 1$ & $0.000000 e+00$ & $1.560010 e-02$ & $0 / 1$ & $0.000000 e+00$ & $1.560010 e-02$ \\
\hline
\end{tabular}

Problem 13. Five-diagonal system [19]:

$$
\begin{gathered}
f_{1}(x)=4\left(x_{1}-x_{2}^{2}\right)+x_{2}-x_{3}^{2}, \\
f_{2}(x)=8 x_{2}\left(x_{2}^{2}-x_{1}\right)-2\left(1-x_{2}\right)+4\left(x_{2}-x_{3}^{2}\right)+x_{3}-x_{4}^{2}, \\
f_{i}(x)=8 x_{i}\left(x_{i}^{2}-x_{i-1}\right)-2\left(1-x_{i}\right)+4\left(x_{i}-x_{i+1}^{2}\right)+x_{i-1}^{2} \\
-x_{i-2}+x_{i+1}-x_{i+2}^{2}, \quad i=3,4, \ldots, n-2, \\
f_{n-1}(x)=8 x_{n-1}\left(x_{n-1}^{2}-x_{n-2}\right)-2\left(1-x_{n-1}\right) \\
+4\left(x_{n-1}-x_{n}^{2}\right)+x_{n-2}^{2}-x_{n-3}, \\
f_{n}(x)=8 x_{n}\left(x_{n}^{2}-x_{n-1}\right)-2\left(1-x_{n}\right)+x_{n-1}^{2}-x_{n-2} .
\end{gathered}
$$

Initial guess: $x_{0}=(-2,-2, \ldots,-2)$.

Problem 14. Extended Freudentein and Roth function ( $n$ is even) [20]: for $i=1,2, \ldots, n / 2$

$$
\begin{aligned}
& f_{2 i-1}(x)=x_{2 i-1}+\left(\left(5-x_{2 i}\right) x_{2 i}-2\right) x_{2 i}-13 \\
& f_{2 i}(x)=x_{2 i-1}+\left(\left(1+x_{2 i}\right) x_{2 i}-14\right) x_{2 i}-29 .
\end{aligned}
$$

Initial guess: $x_{0}=(6,3,6,3, \ldots, 6,3)$.
Problem 15. Discrete boundry value problem [21]:

$$
\begin{gathered}
f_{1}(x)=2 x_{1}+0.5 h^{2}\left(x_{1}+t\right)^{3}-x_{2}, \\
f_{i}(x)=2 x_{i}+0.5 h^{2}\left(x_{i}+t i\right)^{3}-x_{i-1}+x_{i+1}, \\
i=2,3, \ldots, n-1, \\
f_{n}(x)=2 x_{n}+0.5 h^{2}\left(x_{n}+t n\right)^{3}-x_{n-1}, \\
t=\frac{1}{n+1} .
\end{gathered}
$$

Initial guess: $x_{0}=(t(t-1), t(2 t-1), \ldots, t(n t-1))$.

Problem 16. Troesch problem [22]:

$$
\begin{gathered}
f_{1}(x)=2 x_{1}+\varrho h^{2} \sin t\left(\varrho x_{1}\right)-x_{2}, \\
f_{i}(x)=2 x_{i}+\varrho h^{2} \sin t\left(\varrho x_{i}\right)-x_{i-1}-x_{i+1}, \\
i=2,3, \ldots, n-1, \\
f_{n}(x)=2 x_{n}+\varrho h^{2} \sin t\left(\varrho x_{n}\right)-x_{n-1}, \\
t=\frac{1}{n+1}, \quad \varrho=10 .
\end{gathered}
$$

Initial guess: $x_{0}=(0,0, \ldots, 0)$.

In the experiments, the parameters in Algorithm 1 and the normal BFGS method were chosen as $r=0.1, \rho=0.5$, $m_{1}=6, \delta_{1}=\delta_{2}=0.001, H_{0}$ and is the unit matrix. All codes were written in MATLAB r2013b and run on PC with 
6600@2.40 GHz Core 2 CPU processor and 4.00 GB memory and Windows 7 operation system. We stopped the program when the condition $\|h(x)\| \leq 10^{-4}$ was satisfied. Since the line search cannot always ensure the descent condition $d_{k}^{T} h_{k}<0$, uphill search direction may occur in the numerical experiments. In this case, the line search rule maybe fails. In order to avoid this case, the stepsize $\alpha_{k}$ will be accepted if the searching time is larger than eight in the inner circle for the test problems. We also stop this program if the iteration number arrived at 1000 . The columns of the tables have the following meaning.

Dim: the dimension. NI: the total number of iterations.

NG: the number of the norm function evaluations. Time: the CPU time in second.

GN: the normal value of $\|h(x)\|$ when the program stops.

$\mathrm{NaN}$ : not-a-number, impling that the code fails to get a real value.

Inf: returning the IEEE arithmetic representation for positive infinity or infinity which is also produced by operations like dividing by zero.

From the numerical results in Table 1, it is not difficult to show that the proposed method is more successful than the normal BFGS method. We can see that there exist many problems which can not be successfully solved by the normal BFGS method. Moreover, the normal BFGS method fails to get real value for several problems. Then we can conclude that the presented method is more competitive than the normal BFGS method.

\section{Conflict of Interests}

The authors declare that there is no conflict of interests regarding the publication of this paper.

\section{Acknowledgments}

The authors would like to thank Professor Gonglin Yuan of Guangxi University for his suggestions in the paper organization and help in the codes of the program, which save them much time to complete this paper. The authors also thank the referees for valuable comments and the editor for suggestions in the idea and the English of this paper, which improves the paper greatly. This work is supported by Guangxi NSF (Grant no. 2012GXNSFAA053002) and China NSF (Grant no. 11261006).

\section{References}

[1] J. M. Ortega and W. C. Rheinboldt, Iterative Solution of Nonlinear Equations in Several Variables, Academic Press, New York, NY. USA, 1970.

[2] P. N. Brown and Y. Saad, "Convergence theory of nonlinear Newton-Krylov algorithms," SIAM Journal on Optimization, vol. 4, no. 2, pp. 297-330, 1994.
[3] D. Zhu, "Nonmonotone backtracking inexact quasi-Newton algorithms for solving smooth nonlinear equations," Applied Mathematics and Computation, vol. 161, no. 3, pp. 875-895, 2005.

[4] G. Yuan and X. Lu, "A new backtracking inexact BFGS method for symmetric nonlinear equations," Computers \& Mathematics with Applications, vol. 55, no. 1, pp. 116-129, 2008.

[5] D. Li and M. Fukushima, "A globally and superlinearly convergent Gauss-Newton-based BFGS method for symmetric nonlinear equations," SIAM Journal on Numerical Analysis, vol. 37, no. 1, pp. 152-172, 1999.

[6] G. Gu, D. Li, L. Qi, and S. Zhou, "Descent directions of quasiNewton methods for symmetric nonlinear equations," SIAM Journal on Numerical Analysis, vol. 40, no. 5, pp. 1763-1774, 2002.

[7] S. G. Nash, "A survey of truncated-Newton methods," Journal of Computational and Applied Mathematics, vol. 124, no. 1-2, pp. 45-59, 2000.

[8] A. Griewank, "The "global" convergence of Broyden-like methods with a suitable line search," Australian Mathematical Society B, vol. 28, no. 1, pp. 75-92, 1986.

[9] G. Yuan and S. Yao, "A BFGS algorithm for solving symmetric nonlinear equations," Optimization, vol. 62, no. 1, pp. 85-99, 2013.

[10] R. H. Byrd, J. Nocedal, and R. B. Schnabel, "Representations of quasi-Newton matrices and their use in limited memory methods," Mathematical Programming, vol. 63, no. 1-3, pp. 129$156,1994$.

[11] G. Yuan, Z. Wei, and S. Lu, "Limited memory BFGS method with backtracking for symmetric nonlinear equations," Mathematical and Computer Modelling, vol. 54, no. 1-2, pp. 367-377, 2011.

[12] G. Yuan, "A new method with descent property for symmetric nonlinear equations," Numerical Functional Analysis and Optimization, vol. 31, no. 7-9, pp. 974-987, 2010.

[13] G. Yuan and X. Li, "A rank-one fitting method for solving symmetric nonlinear equations," Journal of Applied Functional Analysis, vol. 5, no. 4, pp. 389-407, 2010.

[14] G. Yuan, X. Lu, and Z. Wei, "BFGS trust-region method for symmetric nonlinear equations," Journal of Computational and Applied Mathematics, vol. 230, no. 1, pp. 44-58, 2009.

[15] G. Yuan, Z. Wei, and X. Lu, "A BFGS trust-region method for nonlinear equations,” Computing, vol. 92, no. 4, pp. 317-333, 2011.

[16] R. H. Byrd and J. Nocedal, "A tool for the analysis of quasiNewton methods with application to unconstrained minimization," SIAM Journal on Numerical Analysis, vol. 26, no. 3, pp. 727-739, 1989.

[17] M. A. Gomes-Ruggiero, J. M. Martínez, and A. C. Moretti, "Comparing algorithms for solving sparse nonlinear systems of equations," SIAM Journal on Scientific and Statistical Computing, vol. 13, no. 2, pp. 459-483, 1992.

[18] M. Raydan, “The Barzilai and Borwein gradient method for the large scale unconstrained minimization problem," SIAM Journal on Optimization, vol. 7, no. 1, pp. 26-33, 1997.

[19] G. Y. Li, "Successive column correction algorithms for solving sparse nonlinear systems of equations," Mathematical Programming, vol. 43, no. 2, pp. 187-207, 1989.

[20] Y. Bing and G. Lin, "An efficient implementation of Merrill's method for sparse or partially separable systems of nonlinear equations," SIAM Journal on Optimization, vol. 1, no. 2, pp. 206221, 1991. 
[21] J. J. Moré, B. S. Garbow, and K. E. Hillstrom, "Testing unconstrained optimization software," ACM Transactions on Mathematical Software, vol. 7, no. 1, pp. 17-41, 1981.

[22] S. M. Roberts and J. J. Shipman, "On the closed form solution of Troesch's problem," Journal of Computational Physics, vol. 21, no. 3, pp. 291-304, 1976. 


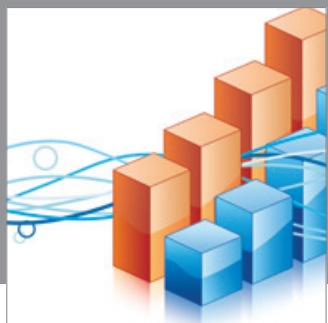

Advances in

Operations Research

mansans

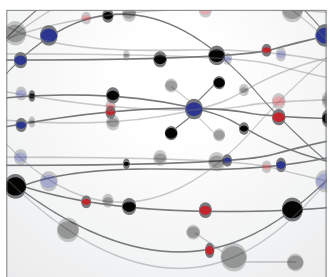

The Scientific World Journal
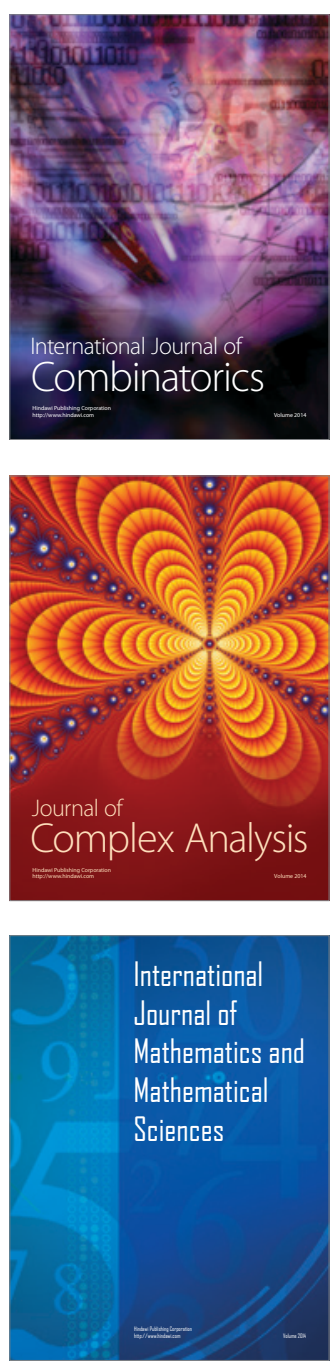
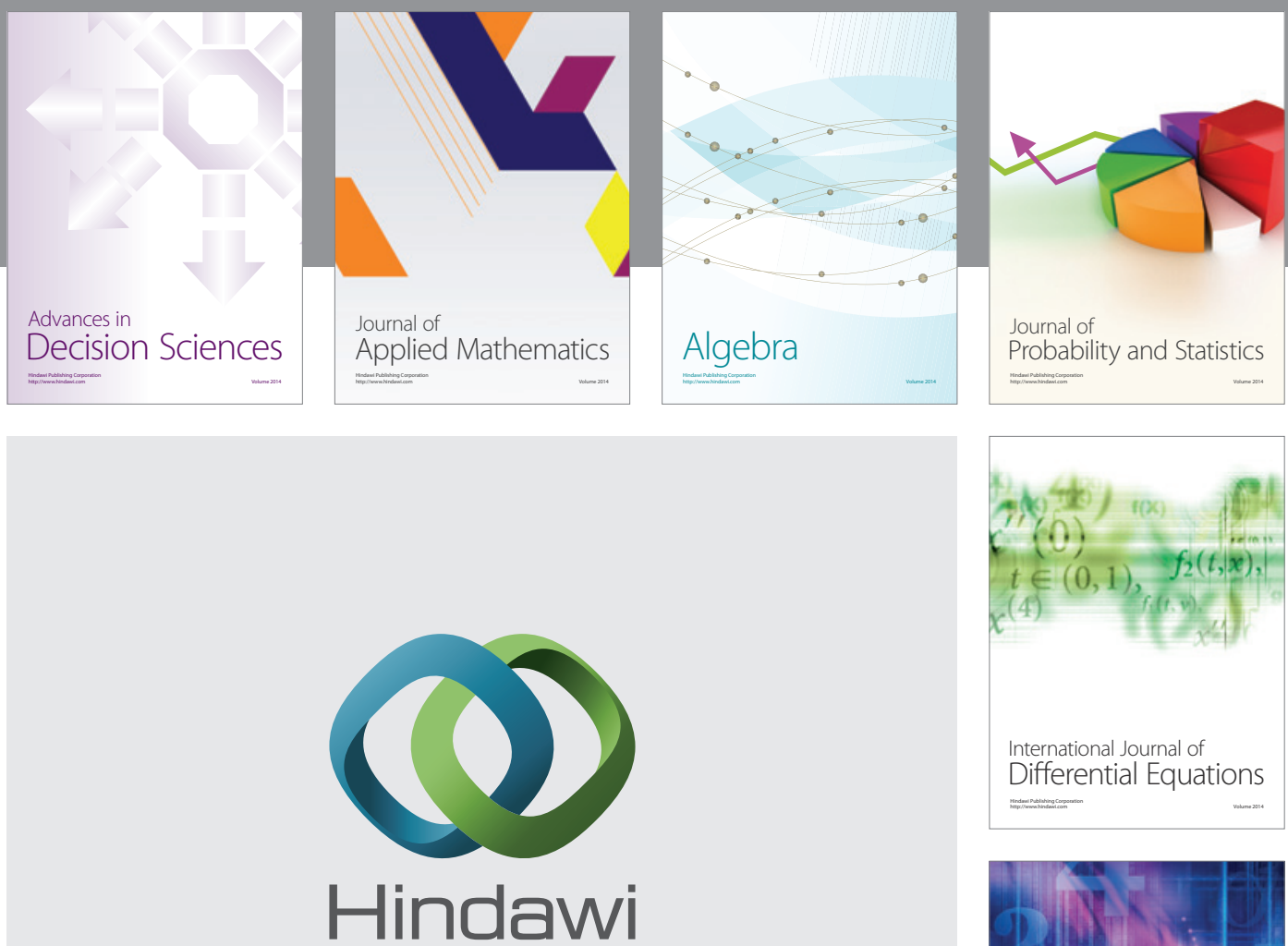

Submit your manuscripts at http://www.hindawi.com
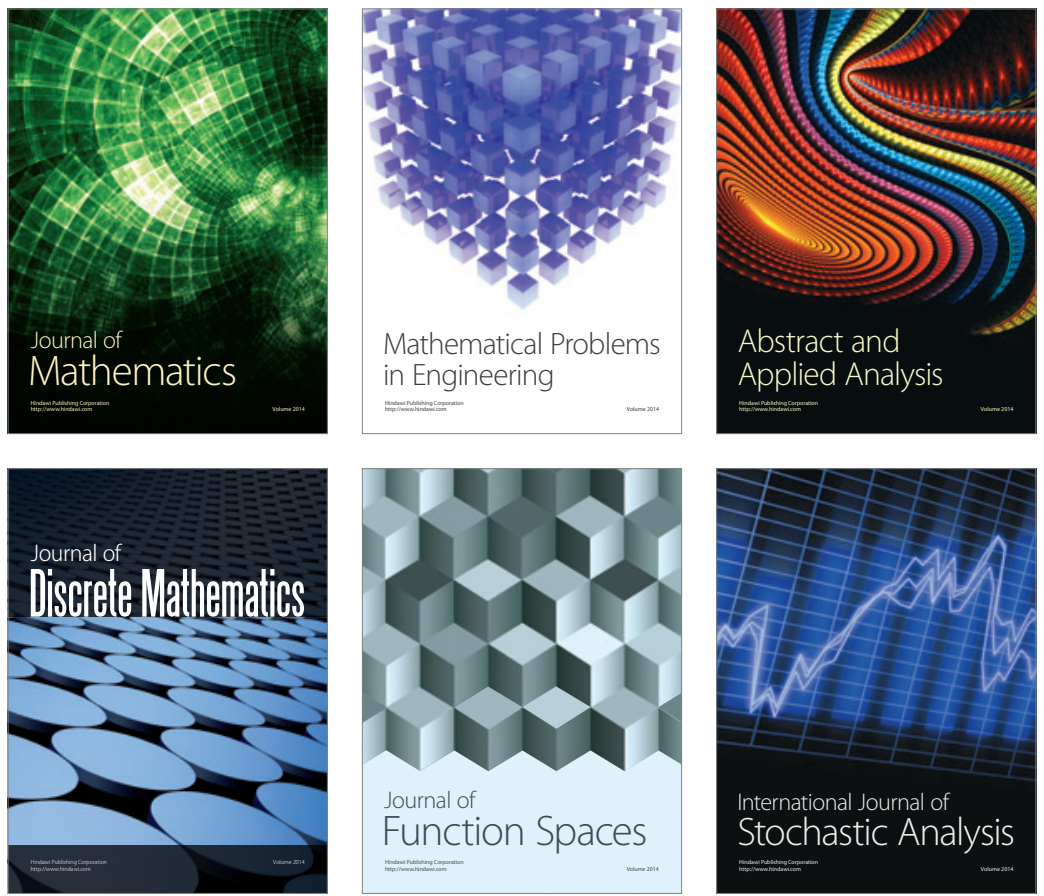

Journal of

Function Spaces

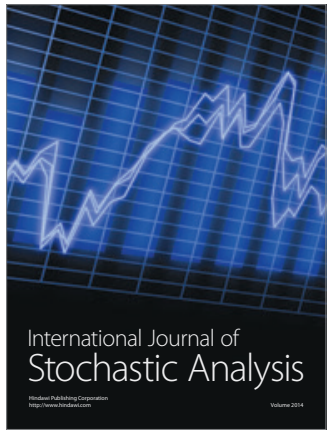

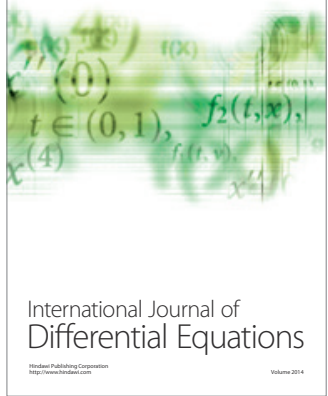
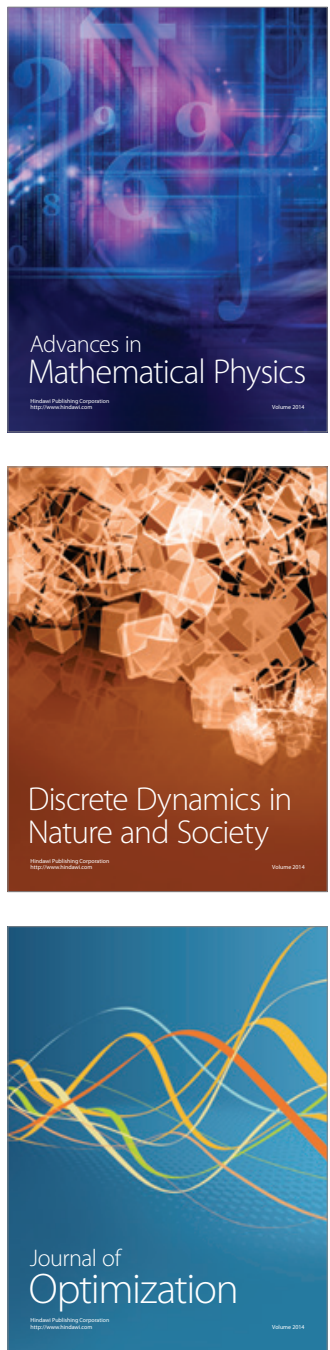